\title{
PREDICTOR BIOMARKERS OF NONELECTIVE HOSPITAL READMISSION AND MORTALITY IN MALNOURISHED HOSPITALIZED OLDER ADULTS
}

\author{
K.M. PENCINA ${ }^{1}$, S. BHASIN ${ }^{1}$, M. LUO², G.E. BAGGS², S.L. PEREIRA², G.J. DAVIS ${ }^{3}$, N.E. DEUTZ4, \\ T.G. TRAVISON ${ }^{5}$
}

\begin{abstract}
1. Research Program in Men's Health: Aging and Metabolism, Boston Claude D. Pepper Older Americans Independence Center, Brigham and Women's Hospital, Harvard Medical School, 221 Longwood Avenue, Boston, MA, USA; 2. Abbott Nutrition Division, Research and Development, 3300 Stelzer Road, Columbus, OH, USA; 3. Abbott Diagnostic Division, Oncology Diagnostics \& Immunoassay Development R\&D, 100 Abbott Park Road, Abbott Park, IL, USA; 4. Center for Translational Research in Aging and Longevity, Department of Health and Kinesiology, Texas A \& M University, College Station, TX, USA; 5. Institute for Aging Research, Hebrew SeniorLife, Department of Medicine, Beth Israel Deaconess Medical Center and Harvard Medical School, Boston, MA, USA.

Corresponding author: Karol M. Pencina, PhD, Research Program in Men’s Health: Aging and Metabolism, Brigham and Women's Hospital, 221 Longwood Avenue, Boston, MA 02115 Phone: 617525 9049, Email: kpencina@bhw.harvard.edu
\end{abstract}

\begin{abstract}
Background: 90-day mortality and rehospitalizations are important hospital quality metrics. Biomarkers that predict these outcomes among malnourished hospitalized patients could identify those at risk and help direct care plans. Objectives: To identify biomarkers that predict 90-day (primary) and 30-day (secondary) mortality or nonelective rehospitalization. Design and Participants: An analysis of the ability of biomarkers to predict 90- and 30-day mortality and rehospitalization among malnourished hospitalized patients. Setting: 52 blood biomarkers were measured in 193 participants in NOURISH, a randomized trial that determined the effects of a nutritional supplement on 90-day readmission and death in patients $>65$ years. Composite outcomes were defined as readmission or death over 90-days or 30-days. Univariate Cox Proportional Hazards models were used to select best predictors of outcomes. Markers with the strongest association were included in multivariate stepwise regression. Final model of hospital readmission or death was derived using stepwise selection. Measurements: Nutritional, inflammatory, hormonal and muscle biomarkers. Results: Mean age was 76 years, $51 \%$ were men. In univariate models, 10 biomarkers were significantly associated with 90-day outcomes and 4 biomarkers with 30-day outcomes. In multivariate stepwise selection, glutamate, hydroxyproline, tau-methylhistidine levels, and sex were associated with death and readmission within 90-days. In stepwise selection, age-adjusted model that included sex and these 3 amino-acids demonstrated moderate discriminating ability over 90-days (C-statistic 0.68 (95\% CI 0.61, 0.75); age-adjusted model that included sex, hydroxyproline and Charlson Comorbidity Index was predictive of 30-day outcomes (C-statistic 0.76 (95\%CI 0.68, 0.85). Conclusions: Baseline glutamate, hydroxyproline, and tau-methylhistidine levels, along with sex and age, predict risk of 90-day mortality and nonelective readmission in malnourished hospitalized older patients. This biomarker set should be further validated in prospective studies and could be useful in prognostication of malnourished hospitalized patients and guiding in-hospital care.
\end{abstract}

Key words: Biomarkers, 90-day readmission, 30-day readmission, nutritional biomarkers of mortality and readmission, mortality in hospitalized patients.

\section{Introduction}

Twenty percent of patients hospitalized for an acute illness are readmitted nonelectively within 90-days; 90-day nonelective rehospitalizations are associated with poor outcomes and increased healthcare costs (1-3). Recent changes in the Medicare reimbursement policies in the United States and the Provisions in the Patient Protection and Affordable Care Act now consider nonelective readmission rates as an important hospital quality metric $(1,4)$. Therefore, there is substantial interest in developing biomarkers that can predict 90- and 30-day mortality and nonelective readmissions (5-10).

Malnutrition is prevalent in hospitalized older adults and associated with longer length of hospital stay, increased risk for patient readmissions, increased overall mortality, and poor health outcomes, including higher rates of 90- and 30-day nonelective readmissions (11-15). There is an unmet need to identify biomarkers that predict mortality and the risk of readmission in malnourshed hospitalized elderly patients. Such biomarkers could be helpful in identifying high-risk patients upon admission so more intensive interventions could be targeted on these patients early to improve health outcomes and reduce mortality and rehospitalization rates.

Accordingly, the primary aim of this investigation was to Identify biomarkers that predict an increased risk of 90-day mortality or rehospitalization in malnourished older adults admitted to the hospital. A secondary aim was to identify markers that predict an increased risk of hospital readmission or death within 30 days post discharge.

To address these aims, we measured 52 blood biomarkers in the participants in NOURISH (Nutrition Effect On Unplanned Readmissions and Survival in Hospitalized Patients) Trial (18), a randomized trial that determined the effects of a nutritional supplement on 90-day readmission and death in malnourished 


\section{THE JOURNAL OF FRAILTY \& AGING}

hospitalized patients $>65$ years. The relatively large sample size of the NOURISH trial, and the availability of data on readmission and death, and availability of samples for the measurement of a large number of nutritional, inflammatory, hormonal and muscle biomarkers enabled us to test the hypothesis that one or more circulating biomarker can predict the risk of nonelective readmission or death within 90 days and 30 days after hospital discharge among malnourished hospitalized patients.

\section{Methods}

\section{The NOURISH Trial}

The design and primary results of the NOURISH Trial have been published (15, ClinicalTrials.gov Identifier: NCT01626742) and are briefly discussed in Supplementary materials.

\section{The Participants}

As described (15), the eligible participants were 65 years or older and had been hospitalized within the preceding 72 hours with a primary diagnosis of acute myocardial infarction, heart failure, chronic obstructive pulmonary disease, or pneumonia. These conditions were selected because these are four of the commonest causes of hospitalization and nonelective readmission, and major contributors to 30- and 90-day mortality among hospitalized patients. Furthermore, under the Hospital Readmission Reduction Program (HRRP) these are the four nonsurgical conditions that CMS monitors and penalizes hospitals for excess readmission rates.

The nutritional status was assessed using the subjective global assessment (SGA) and those deemed to have moderate (class B) or severe malnutrition (class C) were deemed eligible (15). Main exclusion criteria included diabetes mellitus determined by medical history and $\mathrm{HbA1c}$; currently active or treated cancer; and severe renal or liver dysfunction (15).

The study interventions are described in Supplementary materials. The current analyses aimed to identify baseline biomarkers that were associated with 90 and 30-day outcomes in 193 individuals in whom blood sample was available at admission for biomarker analyses.

\section{Outcomes}

The primary outcome for this secondary analysis was the 90-day composite incidence of nonelective hospital readmission or death post-discharge. The secondary outcome was the occurrence of nonelective hospital readmission or death within 30 days post-discharge.

\section{Biomarkers}

A total of 52 circulating biomarkers were measured at baseline in 193 individuals who participated in the Nourish Trial: these included amino acids and related metabolites, nutritional markers, hormones, acute phase reactants, inflammatory markers, minerals and others. The biomarkers that were included in the analyses are listed in Table 1 and blood sample collection and measurement methods are provided in Supplementary materials.

\section{Statistical Methods}

\section{The Analytic Sample}

The current analysis included all 193 patients having baseline assessments of blood biomarkers. The biomarkers whose concentrations were below the lower limit of quantitation in $\geq 30 \%$ of subjects were excluded from statistical analyses.

\section{Analytical Approach}

Statistical methods implemented in this study are discussed in detail in Supplementary materials. The aim of this study was to identify baseline biomarkers that were associated with and predictive of the outcome: composite 90-day and 30-day nonelective hospital readmission or death post discharge using Cox Proportional Hazards model.

Fifty two baseline markers were divided into 9 clinically meaningful classes of predictors to facilitate selection of the most informative candidates and to avoid potential overfitting: demographic, nutritional, hormones, acute phase proteins, inflammation, minerals, iron related, amino acids and other. Univariate Cox Proportional Hazards models were performed on all analyzed markers to select best predictors within each class. Markers within each class, with the strongest association with outcomes based on p-value, were selected for further consideration. The final model of hospital readmission or death was derived using stepwise selection technique with entry and stay criteria of alpha 0.05 . C-statistics as a metric of model discrimination was calculated to assess performance of the final model. Goodness-of-fit was evaluated quantitively using Greenwood-Nam-D'Agostino calibration test for survival models (17) (p-values larger than 0.05 indicates good calibration) and qualitatively using calibration plots.

Inclusion into the final model of treatment assignment, age and other important clinical variables related to outcome, was also considered and those models were compared to model derived from stepwise selection. To correct for overfitting, final model performance was internally validated by computation of bootstrap estimate of optimization for C-statistic (18).

\section{Results}

The analyses included all 193 participants, who had measurement of biomarkers at baseline.

\section{Baseline Characteristics of Study Participants}

The participants in the analytic sample were on average 76 years old, $51 \%$ were men, and the mean body mass index was 


\section{PREDICTORS OF READMISSION AND MORTALITY}

$24.7 \mathrm{~kg} / \mathrm{m} 2$ (Supplementary Table 1). Overall, the participants in both treatment arms had similar baseline characteristics. Similar to parent trial, the Kaplan-Meier estimates for readmission and death outcomes did not differ between placebo and treatment groups, in our analytical cohort, with Log-rank and Tarone-Ware tests being non-significant (all p-values > $0.20)$ (data not shown).

Table 1

Results from stepwise selection for 90-day and 30-day outcome $(\mathrm{N}=191)$

\begin{tabular}{lcc}
\hline 90-day outcome & Hazard Ratio $(\mathbf{9 5 \%}$ CIs $)$ & P-value \\
\hline Sex, Female & $0.56(0.31,1.00)$ & 0.048 \\
Glutamate, $23,4 \mu \mathrm{M}$ & $0.64(0.44,0.94)$ & 0.021 \\
Hydroxyproline, $6.2 \mu \mathrm{M}$ & $1.29(1.00,1.66)$ & 0.049 \\
Tau-methylhistidine, $3.2 \mu \mathrm{M}$ & $1.31(1.07,1.59)$ & 0.007 \\
\hline 30-day outcome & Hazard Ratio $(\mathbf{9 5 \%}$ CIs) & P-value \\
\hline Sex, Female & $0.22(0.07,0.65)$ & 0.006 \\
Total Charlson Comorbidity Score & $1.35(1.05,1.72)$ & 0.017 \\
Hydroxyproline, $6.2 \mu \mathrm{M}$ & $1.45(1.06,2.00)$ & 0.021 \\
\hline
\end{tabular}

Legend: * Hazard Ratio estimates and 95\% CIs are expressed per one interquartile range. C-statistics for 90-day outcome model selected in stepwise procedure was 0.68 (95\% CI: $0.61,0.75)$. C-statistic for 30-day outcome model selected in stepwise procedure was $0.76(95 \% \mathrm{CI}: 0.68,0.84)$

\section{Figure 1}

Hazard Ratios for 90-day outcome. Predictors selected in the stepwise regression model

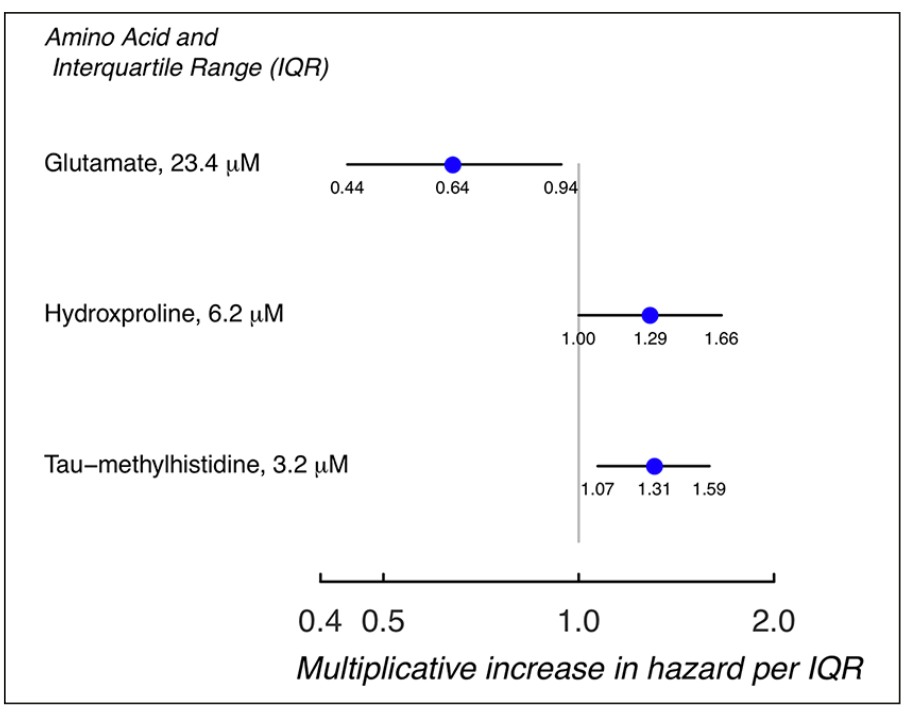

\section{Model selection}

We first performed univariate Cox proportional hazard models on all analyzed biomarkers to select the predictors within each class that were significantly associated with 90-day and 30-day outcomes. In the univariate analyses, the following 10 biomarkers were significantly associated with the 90-day nonelective hospital readmission or death at the $<0.05$ level: sex, Charlson Comorbidity Index score, parathyroid hormone (PTH), serum amyloid A (SAA), serum amyloid P component (SAP), ferritin, glutamate, hydroxyproline, citrulline and taumethylhistidine (Supplementary Table 2).

In the univariate analyses of nonelective hospital readmission or death within 30 days post discharge, only 4 biomarkers were below the 0.05 significance level and all 4 were included in the stepwise selection model: sex, Charlson Comorbidity Index score, glutamate and hydroxyproline (Supplementary Table 3).

In multivariate stepwise selection, sex and circulating levels of the amino acids glutamate, hydroxyproline and taumethylhistidine were associated with death and nonelective hospital readmission within 90-days post-discharge (Table 1). Although not statistically significant, age was included in the final model because of its clinical import. Glutamate, hydroxyproline and tau-methylhistidine demonstrated statistically significant association with the primary outcome of 90-day death or nonelective hospital readmission with hazard ratios expressed per one interquartile range difference of 0.64 (95\% CIs: 0.44, 0.94; p-value=0.021), 1.29 (95\% CIs: 1.0,1.66, p-value $=0.049)$, and 1.31 (95\% CIs: 1.07, 1.59; p-value $=0.007)$, respectively, indicating higher glutamate levels were associated with fewer events, and higher tau-methylhistidine or hydroxyproline levels associated with more events in this population (Figure 1).

Table 2

Multivariate Cox Proportional Hazards regression model with performance metrics for 90-day incidence of hospital readmission or death post discharge

\begin{tabular}{|c|c|c|c|}
\hline Model & $\begin{array}{c}\text { Hazard Ratio } \\
\text { (95\% CIs) }\end{array}$ & P-value & $\begin{array}{l}\text { C-statistics } \\
\text { (95\% CIs) }\end{array}$ \\
\hline Age, 10 years & $0.89(0.60,1.31)$ & 0.55 & $0.59\left(0.51,0.67^{*}\right.$ \\
\hline Sex, Female & $0.58(0.32,1.04)$ & 0.07 & \\
\hline Glutamate, $23.4 \mu \mathrm{M}$ & $0.64(0.44,0.92)$ & 0.017 & $0.68(0.61,0.75)^{* * *}$ \\
\hline Hydroxyproline, $6.2 \mu \mathrm{M}$ & $1.24(0.94,1.64)$ & 0.12 & \\
\hline Tau-methylhistidine, $3.2 \mu \mathrm{M}$ & $1.24(1.00,1.53)$ & 0.047 & \\
\hline Total Charlson Comorbidity Score & $1.14(0.95,1.38)$ & 0.17 & $0.69(0.62,0.76)^{* * * *}$ \\
\hline Treatment & $0.80(0.45,1.42)$ & 0.44 & $0.69(0.62,0.76)^{\dagger}$ \\
\hline \multicolumn{4}{|c|}{$\begin{array}{l}\text { Legend: Glutamate, Hydroxyproline, Tau-methylhistidine per interquartile range. } \\
* \text { C-statistic for model with Age and Sex only. ** C-statistic for model with Age, } \\
\text { Sex, Glutamate, Hydroxyproline, and Tau-methylhistamine. *** C-statistic for model } \\
\text { with Age, Sex, Glutamate, Hydroxyproline, Tau-methylhistamine, and Total Charlson } \\
\text { Comorbidity Score. } \dagger \text { C-statistic for model with Age, Sex, Glutamate, Hydroxyproline, } \\
\text { Tau-methylhistamine, Total Charlson Comorbidity Score and Intervention. }\end{array}$} \\
\hline
\end{tabular}

For 30-day outcomes, for the reasons discussed above, the age factor was added to the final risk model. In the stepwise selection three biomarkers, sex, Charlson Comorbidity Index score and Hydroxyproline stayed in multivariate model and showed statistically significant association with 30-day nonelective rehospitalization and death post-discharge with HRs of 0.22 (95\% CIs: 0.07, 0.65; p-value $=0.006), 1.35(95 \%$ 
CIs: 1.05, 1.72; p-value $=0.017)$ and 1.45 (95\% CIs: 1.06, 2.00; $\mathrm{p}=0.021$ ), respectively (Table 1$)$.

\section{Model performance}

Model discrimination. Final selected multivariate regression model for 90-day nonelective hospital readmission or death demonstrated moderate ability of biomarkers to distinguish between events and non-events (Table 2). Adding amino acid biomarkers (glutamate, hydroxyproline and tau-methylhistidine) to the model improved the discrimination in comparison with model with age and sex only (C-statistic: 0.68 (95\% CI: 0.61, 0.75)). The inclusion of the Charlson Comorbidity Index score only slightly improved model performance of the multivariate model (C-statistic: 0.69 (95\% CIs: 0.62, 0.76)). Inclusion of the intervention factor in the model did not affect the discrimination metrics (Table 2).

Similar analysis was done for the 30-day outcome. In the multivariate stepwise method, the age-adjusted model that included sex, Charlson Comorbidity Index score and Hydroxyproline demonstrated good discrimination with C-statistic 0.76 (95\% CIs: 0.68, 0.85). Further addition of intervention factor into the model did not improve the C-statistics: 0.77 (95\% CIs: 0.69, 0.85) (Table 3).

\section{Table 3}

Multivariate Cox Proportional Hazards regression model with performance metrics for 30-day incidence of hospital readmission or death post discharge

\begin{tabular}{lccc}
\hline \multicolumn{1}{c}{ Model } & $\begin{array}{c}\text { Hazard Ratio } \\
(\mathbf{9 5 \%} \text { CIs })\end{array}$ & P-value & $\begin{array}{c}\text { C-stat } \\
(\mathbf{9 5 \%} \text { CIs })\end{array}$ \\
\hline Age, 10 years & $0.95(0.52,1.71)$ & 0.85 & $0.67(0.56,0.79)^{*}$ \\
Sex, Female & $0.22(0.07,0.65)$ & 0.006 & \\
Charlson Comorbidity Index score & $1.38(1.06,1.79)$ & 0.018 & $0.76(0.68,0.85)^{* *}$ \\
Hydroxyproline, $6.2 \mu \mathrm{M}$ & $1.47(1.05,2.08)$ & 0.027 & \\
Treatment & $1.45(0.61,3.44)$ & 0.39 & $0.77(0.69,0.85)^{* * *}$ \\
\hline $\begin{array}{l}\text { Legend: * C-statistic for model with Age and Sex only. ** C-statistic for model with } \\
\text { Age, Sex, Charlson Comorbidity Score and Hydroxyproline. *** C-statistic for model } \\
\text { with Age, Sex, Charlson Comorbidity Score, Hydroxyproline and intervention. }\end{array}$
\end{tabular}

Model calibration. Age-adjusted models selected by the stepwise procedure for both outcomes demonstrated acceptable calibration (Supplementary Figures 1 and 2). Furthermore, goodness-of-fit metric demonstrated good calibration for the age-adjusted model with sex indicator and three amino acids for 90-day hospital readmission or death (Chi-squared $=5.16$, $\mathrm{p}=0.271$ ).

Internal Validation. C-statistic metrics corrected for Harrell's bootstrap estimate for optimization were 0.65 (95\% CI: 0.58 , $0.72)$ and $0.73(95 \% \mathrm{CI}: 0.64,0.81)$ for stepwise selected age-adjusted models for the 90-day and the 30-day outcomes, respectively.

\section{Discussion}

In malnourished hospitalized patients, the baseline circulating levels of glutamate, hydroxyproline, and taumethylhistidine, when considered together with sex and age were predictive of the risk of 90-day mortality and nonelective hospital readmission. These findings are clinically important because identifying hospitalized, malnourished patients at risk of death and nonelective rehospitalization could help target additional interventions and resources towards this at-risk population. Such prognostic information offered by this biomarker set could inform the care plan and its intensity for hospitalized patients. These findings should be validated in a prospective study of similar hospitalized older patients.

The circulating biomarker amino acids - hydroxyproline, tau-methylhistidine, and glutamate - that emerged from the multivariate step wise regression analyses as predictors of the primary outcome have biologic plausibility. Cachexia observed in sepsis and other catabolic states is associated with increased breakdown of myofibrillar proteins (19-21). Mechanistically, acute illnesses are associated with increased muscle protein breakdown, mediated typically by upregulation of proteins involved in protein degradation, such as polyubiquitins, $\mathrm{Ub}$ fusion proteins, the Ub ligases atrogin-1/MAFbx (muscle atrophy f box) and MuRF-1 (muscle-specific RING finger-1), multiple subunits of $20 \mathrm{~S}$ proteasome and its $19 \mathrm{~S}$ regulator, and cathepsin L (19-21). Urinary excretion of tau-methylhistidine and its circulating levels have been used as a biologic marker for skeletal muscle protein breakdown in humans and animals (22-23). Similarly, hydroxyproline is a component of collagen, and increases in serum hydroxyproline levels suggest increased collagen turnover, implicating musculoskeletal remodeling. Glutamate levels are low in sepsis and acute illness and glutamate metabolism is altered in illness wherein deamination predominates over transamination (24). Consequently, circulating glutamine levels are reduced in patients with critical illness or following major surgery, and a low plasma concentration of both glutamate (24) and glutamine (25-26) at ICU admission has been recognized as an independent risk factor for post-ICU mortality. Old age has been well recognized as a risk factor for adverse health outcomes among hospitalized patients $(3,27)$. The role of sex as a predictor of health outcomes in hospitalized patients is complex, less well understood, and varies with disease condition. Among middle-aged adults hospitalized with an acute myocardial infarction, women have higher unadjusted mortality rates than men; however, in general, overall mortality rates are higher among older hospitalized men than among older women $(3,27)$.

A number of additional biomarkers, including albumin, prealbumin, transferrin, C-reactive protein, creatinine, cystatin 3 , pentraxine-3, hemoglobin, red cell distribution width, fibrinogen, and others have been evaluated individually or in small sets in patients with sepsis, heart failure, acute renal failure, cardiac shock, or in patients admitted to the intensive 


\section{PREDICTORS OF READMISSION AND MORTALITY}

care unit as biomarkers of health outcomes, such as death, intubation, or readmission (5-10). Some of the traditional biomarkers of nutritional status such as albumin and prealbumin did not emerge as predictors in this analysis; this is consistent with recent evidence that these acute phase proteins do not consistently change with weight loss or illness, and their utility as indicators of malnutrition is limited (28).

This study has several strengths and some limitations. The present analyses represent a comprehensive systematic investigation of one of the largest batteries of biomarkers in malnourished hospitalized patients. In contrast to several previous studies that have focused on single or a small set of narrowly-focused biomarkers, we investigated a large number of nutritional, inflammatory, hormonal, and musclerelated circulating biomarkers. We focused on biomarkers for which analytically verified assays are commercially available to facilitate accelerated translation to clinical practice. The primary outcome - a composite 90-day mortality and nonelective rehospitalization post-discharge - is a highly impactful health outcome that has important clinical and public health implications for patient care and healthcare policy. The candidate conditions - acute myocardial infarction, heart failure, chronic obstructive pulmonary disease and pneumonia represent four of most common causes of hospitalization and the most frequent causes of nonelective hospital admission.

The study also has some limitations. The analyses were performed on a sample enrolled in a randomized clinical trial in patients in whom biomarker data were available. The patient population included in these analysis were malnourished because those patients are at high risk of 90-day mortality and readmission. Additional studies are needed in patients who are not malnourished. Some of the traditional markers of nutritional status such as albumin and prealbumin did not emerge as predictors in this analysis; this is consistent with recent evidence that these acute phase proteins do not consistently change with weight loss, illness, or nitrogen balance, and their utility as indicators of malnutrition is limited (28). Also, the temporality of the design and robustness to covariate control suggest the possibility of causal associations between biomarkers and our endpoints; additional larger studies are needed to formally investigate mediation of effects along specific pathways. The relatively small number of mortality events especially during the 30-day postdischarge period, may have limited the statistical power for the analyses of 30-day outcomes. The C-statistics for the primary model for 90-day readmission or death approached the threshold of 0.7 that is generally considered "good" discrimination (29); the ability of our model to distinguish between events and non-events was similar or better than performance reported in other studies with similar settings evaluating hospital readmission outcomes (30). The findings need confirmation in a larger prospective study.

In summary, the baseline circulating levels of the amino acids glutamate, hydroxyproline, and tau-methylhistidine, when considered together with sex and age were predictive of the risk of 90-day mortality and nonelective hospital readmission in malnourished patients who were hospitalized for acute myocardial infarction, heart failure, chronic obstructive pulmonary disease or pneumonia. Additional prospective studies are needed to validate the performance characteristics of this biomarker set in predicting 90-day mortality, nonelective readmission, and other health outcomes. If validated, such a biomarker set could be useful in prognostication of malnourished hospitalized patients, thereby serving as an additional guide to the design and intensity of in-hospital management of hospitalized patients.

Funding support: This investigator-initiated study was supported by funding from Abbott Nutrition to Brigham and Women's Hospital. Dr. Shalender Bhasin reports receiving research grants from NIH, FNIH, AbbVie, Alivegen, FPT, and MIB for investigated-initiated research not related to this manuscript; these grants and contracts are managed by Brigham and Women's Hospital. He has received consulting fees from AbbVie and OPKO. He holds equity interest in FPT, LLC. Drs. Pereira, Luo, Davis and Briggs are employees of Abbott Nutrition and they had the role in interpretation of data and review of the manuscript.

Acknowledgements: We would like to thank Jeff Nelson, $\mathrm{PhD}$ (Abbott Nutrition) for conducting the NOURISH study and providing consultation and support to the project.

Ethical standards: The NOURISH protocol was approved by the Copernicus Group Institutional Review Board for Human Subjects Research (Durham, NC), Western Institutional Review Board for Human Subjects Research (Olympia, WA) and the local Institutional Review Boards of the participating centers. All participants provided written informed consent and were randomized within 72 hours of hospital admission. Drs. Pencina and Travison had full access to the data and performed the analyses. Drs. Pencina, Travison and Bhasin wrote the manuscript; all other authors participated in the review and revisions of the manuscript. Drs. Pereira, Luo, Baggs, and Davis are employees of Abbott Nutrition. They provided the data from the NOURISH Trial and participated in the discussions, review of the analyses and the manuscript.

Open Access: This article is distributed under the terms of the Creative Commons Attribution 4.0 International License (http://creativecommons.org/licenses/by/4.0/), which permits use, duplication, adaptation, distribution and reproduction in any medium or format, as long as you give appropriate credit to the original author(s) and the source, provide a link to the Creative Commons license and indicate if changes were made.

\section{References}

1. Jencks S, Williams M, Coleman E. Rehospitalizations among patients in the Medicare fee-for-service program. N Engl J Med. 2009;360:1418-1428.

2. Hua M, Gong MN, Brady J, Wunsch H. Early and late unplanned rehospitalizations for survivors of critical illness. Crit Care Med. 2015 Feb;43(2):430-8.

3. Marcantonio ER, McKean S, Goldfinger M, Kleefield S, Yurkofsky M, Brennan TA. Factors associated with unplanned hospital readmission among patients 65 years of age and older in a Medicare managed care plan. Am J Med. 1999 Jul;107(1):13-7.

4. Navarro AE, Enguídanos S, Wilber KH. Identifying risk of hospital readmission among Medicare aged patients: an approach using routinely collected data. Home 


\section{THE JOURNAL OF FRAILTY \& AGING}

Health Care Serv Q. 2012:31(2):181-95.

5. Pietro Caironi, Serge Masson, Tommaso Mauri, Barbara Bottazzi, Roberto Leone, Michela Magnoli, Simona Barlera, Filippo Mamprin, Andrea Fedele, Alberto Mantovani, Gianni Tognoni, Antonio Pesenti, Luciano Gattinoni, Roberto Latini, for the ALBIOS Biomarkers Study Investigators Pentraxin-3 in patients with severe sepsis or shock: the ALBIOS trial. Eur J Clin Invest. 2017 Jan; 47(1): 73-83. Published online 2016 Dec 14. doi: 10.1111/eci.12704

6. Ravn B, Prowle JR, Mårtensson J, Martling CR, Bell M. Superiority of Serum Cystatin C Over Creatinine in Prediction of Long-Term Prognosis at Discharge From ICU. Crit Care Med. 2017 Sep;45(9):e932-e940.

7. Spoorenberg SM, Vestjens SM, Rijkers GT, Meek B, van Moorsel CH, Grutters JC, Bos WJ; Ovidius Study Group. YKL-40, CCL18 and SP-D predict mortality in patients hospitalized with community-acquired pneumonia. Respirology. 2017 Apr;22(3):542-550.

8. Ramírez P, Ferrer M, Martí V, Reyes S, Martínez R, Menéndez R, Ewig S, Torres A. Inflammatory biomarkers and prediction for intensive care unit admission in severe community-acquired pneumonia. Crit Care Med. 2011 Oct;39(10):2211-7.

9. Porfyridis I, Georgiadis G, Vogazianos P, Mitis G, Georgiou A. C-reactive protein, procalcitonin, clinical pulmonary infection score, and pneumonia severity scores in nursing home acquired pneumonia. Respir Care. 2014 Apr;59(4):574-81.

10. Niedziela JT, Hudzik B, Szygula-Jurkiewicz B, Nowak JU, Polonski L, Gasior M, Rozentryt P. Albumin-to-globulin ratio as an independent predictor of mortality in chronic heart failure. Biomark Med. 2018 Jun 5. doi: 10.2217/bmm-2017-0378.

11. Sullivan Dh SSWRC. Protein-energy undernutrition among elderly hospitalized patients: A prospective study. JAMA. 1999;281(21):2013-2019

12. Mogensen KM, Horkan CM, Purtle SW, Moromizato T, Rawn JD, Robinson MK, Christopher KB. Malnutrition, Critical Illness Survivors, and Postdischarge Outcomes: A Cohort Study. JPEN J Parenter Enteral Nutr. 2017 May 1:148607117709766.

13. Milne AC, Avenell A, Potter J. Meta-analysis: protein and energy supplementation in older people. Ann Intern Med. Jan 3 2006;144(1):37-48.

14. Schilp J, Bosmans JE, Kruizenga HM, Wijnhoven HA, Visser M. Is dietetic treatment for undernutrition in older individuals in primary care cost-effective? Journal of the American Medical Directors Association. Mar 2014;15(3):226 e227-226 e213.

15. Deutz NE, Matheson EM, Matarese LE, Luo M, Baggs GE, Nelson JL, Hegazi RA, Tappenden KA, Ziegler TR; NOURISH Study Group. Readmission and mortality in malnourished, older, hospitalized adults treated with a specialized oral nutritional supplement: A randomized clinical trial. Clin Nutr. 2016 Feb;35(1):18-26.

16. Wild D. The Immunoassay Handbook, third edition (2005). Chapter 34 by Frank Quinn. Publisher: Elsevier.

17. Demler OV, Paynter NP and Cook NR. Tests of calibration and goodness-of-fit in the survival setting. Stat Med. 2015; 34: 1659-1680.
18. Harrell, F. E., Lee, K. L., \& Mark, D. B (1996). Tutorial in Biostatistics: Multivariable prognostic models. Statistics in Medicine, 15:361-387.

19. Lecker SH, Jagoe RT, Gilbert A, Gomes M, Baracos V, Bailey J, Price SR, Mitch WE, Goldberg AL. Multiple types of skeletal muscle atrophy involve a common program of changes in gene expression. FASEB J. 2004 Jan;18(1):39-51.

20. Wyke SM, Russell ST, Tisdale MJ. Induction of proteasome expression in skeletal muscle is attenuated by inhibitors of NF-kappaB activation. Br J Cancer. 2004 Nov 1;91(9):1742-50.

21. Rutten EP, Franssen FM, Engelen MP, Wouters EF, Deutz NE, Schols AM. Greate whole-body myofibrillar protein breakdown in cachectic patients with chronic obstructive pulmonary disease. Am J Clin Nutr. 2006 Apr;83(4):829-34.

22. Long CL, Muscle protein catabolism in the septic patient as measured by 3-methylhistidine excretion. Metabolism. 1981;30:765-76. and Am J Clin Nutr 1977;30:1349-52.

23. Nagasawa, T., F. Yoshizawa and N. Nishizawa. Plasma N tau-methylhistidine concentration is a sensitive index of myofibrillar protein degradation during starvation in rats. Biosci Biotechnol Biochem 1996;60(3): 501-502.

24. Poeze M, Luiking YC, Breedveld P, Manders S, Deutz NE. Decreased plasm glutamate in early phases of septic shock with acute liver dysfunction is an independent predictor of survival. Clin Nutr. 2008;27(4):523-30

25. Oudemans-van Straaten HM, Bosman RJ, Treskes M, van der Spoel HJ, Zandstra DF. Plasma glutamine depletion and patient outcome in acute ICU admissions. Intensive Care Med. 2001;27(1):84-90.

26. Rodas et al, A low plasma concentration of glutamine at ICU admission is an independent risk factor for post-ICU mortality. Clin Sci (Lond). 2012 Jun;122(12):591-7.

27. Ponzetto M, Maero B, Maina P, Giovannino RR, Ciccone F, Laurence Z. Rubenstein LZ, Fabris F. Risk Factors for Early and Late Mortality in Hospitalized Older Patients: The Continuing Importance of Functional Status . The Journals of Gerontology: Series A. 58: M1049-M1054

28. White JV, Guenter P, Jensen G, Malone A, Schofield M: Academy Malnutrition Work Group; ASPEN Malnutrition task Force; ASPEN Board of Directors J Parenter Enteral Nutr. 2012 May;36(3):275-83.

29. Hosmer DW, Lemeshow. Applied Logistic Regression. 2nd ed. Hoboken, NJ: Wiley; 2000

30. Smith LN, Makam AN, Darden D, Mayo H, Das SR, Halm EA, Nguyen OK. Acute myocardial infarction readmission risk prediction models: a systematic review of model performance.Circ Cardiovasc Qual Outcomes. 2018; 11:e03885. doi: 10.1161/ CIRCOUTCOMES. 